\title{
Diacronie
}

Studi di Storia Contemporanea

$N^{\circ} 8,4 \mid 2011$

Le sembianze di Clio: approcci alla storia

\section{Il cimitero la Verbena: cinquant'anni di violenza politica e sociale a Città del Guatemala}

\section{Paolo Grassi}

\section{Q OpenEdition \\ Journals}

Edizione digitale

URL: http://journals.openedition.org/diacronie/3541

DOI: $10.4000 /$ diacronie.3541

ISSN: 2038-0925

Editore

Association culturelle Diacronie

Notizia bibliografica digitale

Paolo Grassi, « II cimitero la Verbena: cinquant'anni di violenza politica e sociale a Città del

Guatemala », Diacronie [Online], № 8, 4 | 2011, documento 5, online dal 29 octobre 2011, consultato il

21 décembre 2020. URL : http://journals.openedition.org/diacronie/3541 ; DOI : https://doi.org/

10.4000/diacronie.3541 


\title{
Diacronie
}

$5 /$

\section{Il cimitero la Verbena: cinquant'anni di violenza politica e sociale a Città del Guatemala}

\author{
Paolo GRASSI *
}

Partendo dall'analisi etnografica di un luogo specifico della capitale guatemalteca - il cimitero del quartiere la Verbena - l'articolo vorrebbe ripercorrere il filo che collega la recente storia del Paese (segnata da un conflitto armato interno durato trentasei anni, terminato solo nel 1996) all'esplosione di una violenza sociale i cui livelli sono attualmente tra i più alti al mondo. La violenza politica e la violenza sociale che hanno investito il Guatemala mostrano elementi di continuità ancora poco sviscerati, la cui esplicitazione rappresenta una sfida interpretativa indispensabile per comprendere alcune delle dinamiche sociali che interessano oggi non solo quel paese.

A volte si può trovare qualcosa.

Si trovano oggetti, stralci di giornale, targhette XX.

Sono cose che ti riportano ad un'altra epoca.

È successo qualcosa di totalmente differente allora...

Anche se oggi c'è un altro tipo di guerra in Guatemala,

però è diversa, cioè, prima era con l'esercito ${ }^{1}$

(Archeologo della Fundación di Antropología Forense de Guatemala, impegnato in un progetto di riesumazione di resti non identificati presso il Cimitero la Verbena, zona 7 - Ciudad de Guatemala)

1 Intervista dell'8 agosto 2011 


\section{XX}

l principio non compresi il senso di quella frase. Mi trovavo a Città del
Guatemala solo da una settimana. Il secondo viaggio che facevo in quel
paese, trenta giorni per impostare la mia successiva ricerca di campo, che
sarebbe iniziata da lì a circa cinque mesi.

Ero seduto su un pick-up grigio, di fianco a C., diacono italiano allora incaricato, insieme ad altri due parroci, della gestione della parrocchia cattolica della Verbena, una delle colonie di zona 7 di Città del Guatemala² .

«Per studiare la violenza, devi partire da dove muoiono i pandilleros ${ }^{3}$ », disse4.

Fu con queste parole che diresse il pick-up grigio oltre l'ingresso del cimitero della colonia, camposanto dove vengono interrati il maggior numero di XX della capitale, ossia i cadaveri non identificati, i morti senza nome.

Alla Verbena ci avevo messo piede per la prima volta nel 2008, come stagista di un progetto della Cooperazione italiana 5 , progetto finanziato anche grazie alla notorietà mediatica dell'area della sua implementazione ${ }^{6}$. La Verbena sorge infatti al confine della grande discarica municipale di Città del Guatemala, un barranco di spazzatura fonte di sostentamento per centinaia di persone. Ogni giorno schiere di guajeros $^{7}$, come $^{2}$ vengono chiamati gli smistatori di rifiuti, accolgono l'arrivo di decine di camion e iniziano la loro selezione: ferro, alluminio, plastica, vetro, carta, cartone, vestiti usati. Gli anziani e le donne riescono ad accaparrarsi i prodotti che valgono meno sul mercato, i giovani maschi quelli più remunerativi. Tutto quello che può essere recuperato viene raccolto e venduto a compratori, che rivendono il materiale a

2 Il Municipio di città del Guatemala (942.368 abitanti) è suddiviso in 25 zone (la 20, 22, e 23 si trovano in municipi confinanti). Ogni zona è suddivisa in colonie, composte a loro volta da un numero variabile di settori.

3 «Banditi».

4 Appunti di campo, 27 agosto 2010.

5 Progetto intitolato: «Protezione e sviluppo dell'infanzia ed adolescenza lavoratrice a Città del Guatemala», realizzato da: DGCS, Municipalità di Città del Guatemala, Università guatemalteche ed italiane, ONG ed Associazioni locali, Federazione dei Municipi centroamericani (FEMICA). Il progetto fu implementato nel corso di tutto il 2008.

${ }^{6} \mathrm{Su}$ come la costruzione e al mantenimento di una rappresentazione mediatica stigmatizzante di una determinante area favorisca anche la concentrazione di risorse economiche destinate a progetti sociali si veda: FAVA, Ferdinando, Lo Zen di Palermo: antropologia dell'esclusione, Roma, Franco Angeli, 2008.

7 Le condizioni lavorative sono pessime, non esistono precauzioni di alcun tipo. Solo nella zona 3 della città ci sono 1.185 persone che lavorano come guajeros. La metà è analfabeta. Molte persone lavorano con famigliari, mogli, figli, sei giorni alla settimana, quattro o otto ore al giorno. DE LEÓN MALDONADO, Alma, Los segregadores de desechos sólidos - guajeros - en la Ciudad de Guatemala, Guatemala, Universidad de San Carlos, 2003. 
fabbriche che a loro volta lo lavorano per rivenderlo a multinazionali del riciclaggio, in un circolo economico schizofrenico, un business pronto ad arricchire realmente solo i pesci più grossi della catena ${ }^{8}$.

La Verbena ricorda alla capitale guatemalteca le sue problematiche maggiori: non solo quelle più direttamente riconducibili alla discarica municipale, come la crisi nella prestazione di alcuni servizi, il deterioramento ambientale, il disfacimento nel mercato dell'impiego e lo sviluppo di un'economia informale; ma anche quelle meno esplicitamente connesse a quel luogo specifico e tuttavia riscontrabili all'interno del quartiere che lo ospita, quali il deficit abitativo (e la conseguente presenza di diversi asentamientos), la polarizzazione sociale, l'aumento dei livelli di violenza urbana9. Tali problematiche hanno favorito pratiche di rigetto e marginalizzazione da parte della classe media e ricca, concretizzatesi in utilizzi gerarchizzati del suolo urbano. Luoghi di segregazione imposta ed auto-segregazione, gli uni accanto agli altri, due città sovrapposte e parallele, slum e arcipelaghi di quartieri extra lusso, colonie residenziali.

Alla Verbena c'ero tornato nell'agosto del 2010, a distanza di due anni. Ero tornato per cercare casa, convinto che non avrei potuto realizzare la mia investigazione se non in un barrio periferico della capitale. Per questo motivo mi trovavo seduto su quel pickup grigio, al fianco di C., cercando di spiegargli il motivo della mia visita e il perché di quella scelta: fare un'etnografia della violenza di Città del Guatemala, a partire dall'analisi dell'utilizzazione di alcuni dei suoi spazi ${ }^{10}$.

Per C. l'associazione violenza-pandillas ${ }^{11}$ fu immediata. È un'associazione comune a Città del Guatemala, riscontrabile in molte narrazioni sul crimine rafforzate dalla cronaca locale. La stampa racconta quotidianamente di una guerra in atto tra le forze dell'ordine e due pandilla rivali, la Mara Salvatrucha e la Pandilla 18, in conflitto per il controllo di porzioni di territorio urbano ${ }^{12}$.

\footnotetext{
8 COPPOLA Paola, «I bambini di Città del Guatemala dalla discarica alle aule scolastiche», $L a$ Repubblica, 7 dicembre 2007.

9 UNIVERSIDAD RAFAEL LANDIVAR, Cultura de Guatemala: reflexiones y aportes, Vol. I, Guatemala Facultad de Arquitectura y Diseño, Campus Central Guatemala, URL, 2006.

${ }_{10}$ Dal mese di gennaio 2011 sto compiendo le mie ricerche in un quartiere periferico della capitale, in un carcere e in una gated community

${ }^{11}$ Letteralmente «bande». Le pandillas, o maras sono raggruppamenti giovanili relativamente stabili, caratterizzati dall'uso di spazi pubblici urbani, che generano modelli identitari influenti sulla vita quotidiana dei suoi membri. DEMOSCOPIA S. A., Maras y Pandillas, Comunidad y Policía en Centroamérica, Guatemala, ASDI, BCIE, 2007.

${ }^{12}$ ERIC, IDESO, IDIES, IUDOP, Maras y pandillas en Centroamèrica, Vol. I, Managua, UCA, 2001.
} 
I numeri sono poco chiari, ma comunque allarmanti. Solo nel 2006 il tasso d'omicidi per 100.000 abitanti era di 47 persone $^{13}$. I giovani rappresentano il settore della popolazione più colpito. Sempre nel 2006, per esempio, 2.840 furono quelli assassinati nel paese, 2.305 dei quali con arma da fuoco ${ }^{14}$. La maggioranza di questi omicidi avviene nella capitale. Uno studio del 2004 della Procuraduría de los Derechos Humanos cita, tra le principali cause nella classifica delle morti violente di adolescenti e giovani tra i 14 e i 29 anni, la morte procurata da mara e tra mara (32,15\% del totale $\left.{ }^{15}\right)$.

Le pandilla rappresentano una causa importante degli alti livelli di violenza riscontrabili nel paese. Una, non l'unica. L'anatomia della violenza guatemalteca dovrebbe includere diversi fenomeni sociali, quali: esecuzioni extragiudiziali perpetrate dalle forze dell'ordine e azioni di cosiddetta pulizia sociale, rapimenti, furti, traffico di droga, tratta di persone, violenza domestica, linciaggi ${ }^{16}$. Di questo la stampa e la retorica politica sembra a volte non accorgersene: le pandilla hanno il merito di fornire un nemico pubblico chiaramente identificabile, un suitable enemy ${ }^{17}$ stigmatizzabile, un capro espiatorio contro cui sfogare le proprie ansie securitarie.

L'associazione di C. (violenza = pandilla) esprimeva quindi una costruzione simbolica condivisa; la visita al cimitero ne sanciva l'esplicitazione: partire da dove la causa di quella violenza ha fine, dove molti di quei giovani, perché non identificati da nessuno, vengono interrati.

\section{I fatti}

Nel 1821 il Guatemala ottiene la propria indipendenza. Da allora, le caratteristiche economiche, culturali e sociali del paese sono state estremamente contraddistinte da strutture gerarchiche ereditate dal periodo coloniale, concretizzatesi in processi di esclusione sociale, razzismo e discriminazione nei confronti della popolazione indigena e dei mestizos impoveriti ${ }^{18}$.

13 PROGRAMA DE SEGURIDAD CIUDADANA Y PREVENCIÓN DE LA VIOLENCIA, Informe estadístico de la violencia en Guatemala, Guatemala, PNUD, 2007.

${ }_{14} \mathrm{CALDH}$, ¿Y la juventud qué?: Estado situacional de los derechos de la juventud en Guatemala, Guatemala, CALDH, 2009.

15 PROCURADURÍA DE LOS DERECHOS HUMANOS DE GUATEMALA, Informe de muertes violentas de niñez, adolescencia y juventud, Guatemala, PDH, 2004.

16 MOSER, O. N. Caroline, «Urban violence and insecurity: an introductory roadmap», in Environment and Urbanization, 16, 2004, pp. 3-16.

17 WACQUANT, Loïc, «Suitable enemies: foreigners and immigrants in the prisons of Europe», in Punishment \& Society, No. 1, October 1999, pp. 215-222.

${ }^{18} \mathrm{CEH}$, Guatemala, memoria del silencio, Guatemala, F\&G editores, 1999. 
Il 1945, anno dell'insediamento al potere di Juan José Arévalo, segna, da questo punto di vista, per molta storiografia locale, un punto di svolta, un periodo rivoluzionario di cambiamento. In realtà il programma di Arévalo è sì riformatore e più attento alle politiche sociali, ma comunque liberista e moderato. In sei anni, sconfiggendo oltre trenta tentativi di golpe di stato, Arévalo, tra l'altro, rinnoverà banche e sistema monetario e regolamenterà in parte il mercato del lavoro ${ }^{19}$.

Nel 1951 gli succederà Jacobo Arbenz Guzmàn. La sua figura è tutt'oggi punto di riferimento dell'immaginario di parte della sinistra guatemalteca. La sua mitologizzazione locale ricorda quella di altri eroi centroamericani: come per Che Guevara, magliette con il suo nome vengono indossate da giovani radicali.

Il programma di governo di Arbenz Guzmán tende all'indipendenza del paese dall'influenza statunitense. Tra le misure adottate, la più importante riguarda sicuramente la riforma agraria ${ }^{20}$. La riforma (Decreto 900) va tuttavia a toccare non solo gli interessi di imprenditori guatemaltechi, ma anche e soprattutto gli interessi degli Stati Uniti, espropriando grandi quantità di terra alla United Fruit Company (attuale Chiquita) ${ }^{21}$.

La causa economica si associa inoltre ad una motivazione politica: il governo guatemalteco viene accusato di simpatie comuniste, pretesto sufficiente per giustificare un intervento armato nel paese. Le alte schiere della chiesa cattolica, dal canto loro, stanno a guardare. Gli Stati Uniti orchestrano l'azione attraverso l'operazione $B P$ Success. 250-300 uomini invadono il Guatemala, entrando dall'Honduras. Un aereo militare colpisce alcuni obiettivi della capitale. Lo stato tenta una controffensiva, ma invano. Nessun'aspra lotta: l'esercito rifiuta di affrontare gli invasori. Si firma il primo luglio il Patto di San Salvador. La presidenza passa nelle mani di Castillo Armas. È il $1954^{22}$.

Castillo Armas (1954 -1957) cancella la riforma agraria, la costituzione, i sindacati e i partiti. Un Comitato Nazionale di Difesa e una Legge Preventiva Penale contro il Comunismo vengono disposti dal suo governo. Con l'appoggio degli Stati Uniti e la loro

19 MUÑOZ, Jorge Lujan, Breve historia contemporanea de Guatemala, México, Fondo de Cultura Economica, 1998.

${ }^{20}$ Il processo di riforma agraria interessa, in epoche diverse, tutta l'America Latina. La prima riforma agraria avviene in Messico, dopo la rivoluzione del 1910. Dopo il 1960 la riforma agraria è auspicata anche da organismi internazionali come la FAO. Spesso i tentativi attuati vengono frustrati dal potere politico che, in modo ambiguo, sostiene le riforme, alla ricerca di consenso e voti, e, al tempo stesso, le limita. STAVENHAGEN, Rodolfo (ed. by), Agrarian problems and peasant movements in Latin America, New York, Anchor Book, 1970.

${ }^{21}$ CHAPMAN, Peter, Bananas: how the United Fruit Company shaped the world, Edinburgh, Canongate books Ltd., 2007.

22 VELÀSQUEZ CARRERA, Eduardo Antonio, (a cargo de), Jacobo Arbenz Guzmán: "El soldado del pueblo", Guatemala, CEUR, USAC, 2008. 
Dottrina di Sicurezza Nazionale, un clima di timore, sfiducia e repressione viene garantito e sostenuto ${ }^{23}$.

Nel 1960 ha inizio la guerrilla. La resistenza e la reazione governativa si istituzionalizzano, il paese viene militarizzato. Le strategie punitive si dispiegano e si diversificano: vengono uccisi leader politici, perseguitate famiglie e intere comunità, attuate deportazioni, torturati e fatti scomparire dissidenti, studenti universitari, intellettuali, giornalisti. Durante gli anni sessanta la repressione si dirige soprattutto contro la popolazione rurale dell'Oriente del paese; durante gli anni settanta si attesta in capitale; durante i primi anni ottanta la politica contrainsurgente diviene vero e proprio terrorismo di stato ${ }^{24}$.

Due eventi critici potrebbero sancire quest'ultima metamorfosi del conflitto: l'occupazione dell'ambasciata di Spagna e l'avvento al potere di Efraín Ríos Montt. Il 31 gennaio 1980 un gruppo di contadini k’iche ${ }^{25}$ s'impossessa, in segno di protesta contro le arbitrarie espropriazioni delle terre e l'oppressione governativa, dell'ambasciata di Spagna nella capitale. La polizia schiaccia la rivolta incendiando l'edificio, provocando la morte di 39 persone ${ }^{26}$. L'evento desta l'attenzione internazionale, ma il dispositivo contrainsurgente dello Stato non si arresta. Il governo dell'allora presidente Romeo Lucas García continua la propria politica violenta e brutale.

Il 23 marzo 1982 ottiene il potere, con l'ennesimo colpo di stato, Efraín Ríos Montt. Durante il suo breve governo (verrà deposto l'8 agosto 1983) il Guatemala attraverserà uno dei periodi più bui della propria storia. Viene implementata una strategia antisovversiva integrale in grado di combinare aspetti militari, politici, psicosociali e antropologici. Per alimentare il meccanismo del terrore vengono, tra l'altro, potenziati i servizi di intelligence e spionaggio, istituzionalizzate le cosiddette Pattuglie di Autodifesa Civile (PAC) - ronde che prevedevano l'arruolamento forzato della popolazione, creati «villaggi modello» (aree rurali militarizzate per il controllo totalitario dei suoi residenti), formati squadroni paramilitari ${ }^{27}$.

23 VELASQUEZ RIVERA, Edgar de Jesús, «Historia de la Doctrina de Seguridad Nacional», in Convergencia: revista de ciencias sociales, México, Universidad Autónoma del Estado de México, 2002.

${ }^{24}$ REMHI, Guatemala nunca más: informe del proyecto interdiocesano de recuperación de la memoria histórica, Guatemala, ODHAG, 1998.

25 Nome di uno dei gruppi maya del Guatemala. La popolazione indigena nel paese rappresenta circa il 40\% della popolazione totale (K'iche 9.1\%, Kaqchikel 8.4\%, Mam 7.9\%, Q'eqchi 6.3\%, other Mayan 8.6\%, indigeni non-Maya 0.2\%, altri 0.1\%: CIA, The worldfact book, Guatemala). ${ }^{26}$ AGENCIA ACAN EFE, «Demandan juicio por matanza en 1980 en embajada de España», Prensa Libre, 31/01/2011.

27 AGUILERA PERALTA, Gabriel, ROMERO IMERY, George, Dialéctica del terror en Guatemala, San José Costa Rica, EDUCA, 1981. 
Con la caduta di Ríos Montt ${ }^{28}$, inizia un lento processo di pacificazione, a partire dallo smantellamento di tribunali speciali e dalla sostituzione di alcuni membri del parlamento. Anche le forze rivoluzionarie cambiano la loro strategia d'azione. Nel febbraio del 1982 i quattro fronti guerriglieri allora attivi ${ }^{29}$ si uniscono, al principio solo formalmente, poi via via più effettivamente, nella URNG (Unidad Revolucionaria Nacional Guatemalteca) ${ }^{30}$.

Le negoziazioni tra URNG e governo centrale si concluderanno solo quattordici anni dopo. Bisognerà aspettare fino al 29 dicembre 1996, sotto la presidenza di Álvaro Arzú, per vedere firmati definitivamente gli accordi di pace ${ }^{31}$.

\section{La struttura del bando}

La paura ha quindi origini lontane. La strategia militare degli anni dittatoriali innalzò la repressione a livello di simbolo assoluto del potere. La politica della violenza puntò alla distruzione del tessuto sociale, della memoria collettiva e delle tradizioni locali $^{32}$. I fantasmi del terrore nati nel periodo della guerra civile oggi resuscitano, invadendo nuove dimensioni sociali. La violenza si dissalda dalle proprie radici prettamente politiche per ferire l'ambito del quotidiano. La violenza determina oggi non solo la vita economica del paese ${ }^{33}$, ma si concretizza in una struttura mentale e collettiva, una cornice all’interno della quale è costretta e dalla quale significa la propria azione.

La violenza politica precedente al 1996 non può essere disgiunta dalla violenza contemporanea che ha investito il Guatemala. Alcuni degli XX del cimitero la Verbena sono testimoni inconsapevoli di questo processo, vittime dei due tipi di brutalità descritti. Gli ossari generali materializzano fisicamente, attraverso la loro stratigrafia, questo continuum che lega la violenza politica a quella sociale.

28 Ríos Montt è tutt'ora un politico attivo in Guatemala. Fondatore del partito Fronte Repubblicano Guatemalteco, membro del parlamento, provò senza successo, a più riprese (1990, 1995, 2003), a presentare la propria candidatura come presidente del paese.

29 I quattro fronti guerriglieri allora attivi erano: FAR - Fuerzas Armadas Rebeldes, EGP Ejército Guerrillero de los Pobres, ORPA - Organización del Pueblo en Armas e PGT-NDN Nucleo de Dirección Nacional del Partido Guatemalteco del Trabajo.

${ }^{30} \mathrm{CEH}$, Guatemala, memoria del silencio, Guatemala, F\&G editores, 1999.

${ }^{31}$ SECRETARÍA DE LA PAZ, PRESIDENCIA DE LA REPÚBLICA, Los acuerdos de paz en Guatemala, Guatemala 2006. Consultabile all'indirizzo:

URL: < http://www.arias.or.cr/publicaciones/FA05035107.pdf > [consultato il 15/10/2011].

$3^{2}$ MAGRASSI, Marco, Guatemala, Napoli, Città Del Sole, 1997.

33 PROGRAMA DE LAS NACIONES UNIDAS PARA EL DESARROLLO, El costo económico de la violencia en Guatemala, Guatemala, PNUD, 2006. 
Ma una volta dimostrata l'esistenza di questo continuum, è possibile spiegarne unitariamente i suoi aspetti, le sue differenti manifestazioni? Ossia, perché esiste questo continuum, quale funzione riprodurrebbe? Si proverà qui di seguito a fornire una risposta circostanziale alla domanda formulata, utilizzando alcune riflessioni proposte dal filosofo italiano Giorgio Agamben riguardo al concetto di potere.

Il giorno del colpo di stato del 1982, il generale Ríos Montt rilascia in una conferenza stampa la seguente dichiarazione:

«Chi solleverà un'arma contro le forze dell'ordine, sarà fucilato. Fucilato e non assassinato!»34.

Questa affermazione, a prima vista senza senso, non può essere in realtà semplicemente liquidata come frutto della mente di un folle dittatore. In quella a prima vista illogica e paradossale sentenza è possibile al contrario individuare la presenza di una configurazione politica originaria, una condizione generale del potere, fattasi storia materiale di uno specifico paese. È ciò che Giorgio Agamben definisce la struttura del potere sovrano35.

Il fucilato-ma-non-assassinato di Ríoss Montt ricorda molto da vicino, nella propria ambiguità, l'homo sacer analizzato dal filosofo italiano. L'homo sacer, figura del diritto romano, persona considerata colpevole di certi delitti collegati alle divinità puniti con l'isolamento dal gruppo, l'uomo che può essere ucciso ma non sacrificato ${ }^{36}$, è per Agamben un concetto limite, la figura primigenia della vita presa nel bando sovrano, ossia l'esclusione originaria che fonderebbe la dimensione politica. Il fucilato-ma-nonassassinato di Ríoss Montt, alla stregua dell'homo sacer, metterebbe allora in luce la struttura caratteristica del potere, fondata sullo stato d'eccezione37. Uno svelamento sicuramente scomodo ad un governo militare e di fatti impedito e neutralizzato attraverso un'organizzata pianificazione.

Le stime delle vittime del conflitto armato guatemalteco sono solo generiche. $\mathrm{Si}$ parla di almeno 200.000 morti, molti dei quali durante i primi anni Ottanta ${ }^{3}$. Ifucilati-ma-non-assassinati iniziarono ad aumentare in maniera direttamente

34 La dichiarazione è filmata da un regista finlandese ed inserita in un suo documentario: WAHLFORSS, Mikael, Panssaroituja Päiväunia [Ensueños Blindados], Finlandia 1983, 50'. Lo spezzone è visibile all'indirizzo internet:

URL: < http://www.youtube.com/watch?v=kI95aYW62R4 > [consultato il 15/10/2011].

35 AGAMBEN, Giorgio, Homo sacer: il potere sovrano e la nuda vita, Torino, Einuadi, 1995

36 «Uomo sacro è, però, colui che il popolo ha giudicato per un delitto; e non è lecito sacrificarlo, ma chi lo uccide, non sarà condannato per omicidio», Ibidem, p. 79.

37 Il potere può proclamare lo stato d'eccezione, qualcosa che si sottrae all'ordine generale, ma che al tempo stesso, lo palesa, ne segna un limite: «...proprio lo stato di eccezione, come struttura politica fondamentale, nel nostro tempo emerge sempre più in primo piano e tende, in ultimo, a diventare la regola», Ibidem, p. 22.

${ }^{38} \mathrm{CEH}$, Guatemala, memoria del silencio, Guatemala, F\&G editores, 1999. 
proporzionale all'ampliamento del dispositivo repressivo statale. Le sparizioni rappresentarono il corollario necessario a questa strategia del terrore. Delle 52.427 violazioni di cui ha raccolto testimonianza il rapporto REMHI (Progetto Interdiocesano per il Recupero della Memoria Storica), 3.893 riguardano scomparse di persone, la maggioranza delle quali avvenute tra il 1980 e il 1983 (3.082 del totale) ${ }^{39}$. La desaparición forzada divenne una pratica programmata per far sì che il silenzio potesse essere preservato, che la struttura del potere potesse essere conservata. I cadaveri dei desaparecidos vennero fatti svanire o abbandonati, nelle strade, in cimiteri clandestini, a nutrire le schiere dei morti senza nome.

Alcuni degli XX della Verbena seppelliti prima del 1996 potrebbero essere vittime di sparizioni forzate. In questo senso, il progetto di riesumazione della Fondazione di Antropologia Forense del Guatemala iniziato nel 2010 rappresenta non solo un'azione di recupero della memoria storica, un gesto informativo, ma anche, implicitamente, un vero e proprio atto politico, uno sforzo per riportare alla luce un sistema di controllo, un dispositivo del potere.

Ma altri XX potrebbero assolvere, in epoca contemporanea, alla stessa funzione, nonostante la presenza di un sistema politico mutato, risultato di un lento processo di democraticizzazione e pacificazione. Sono gli XX indicatimi dal diacono C., i banditi non identificati. È ancora la riflessione di Agamben a fornirci la connessione. Il bandito (inteso dall'autore in senso ampio, come «messo al bando») può essere considerato «fratello»40 dell'homo sacer. Nell'antichità germanica e scandinava il bandito viene escluso dalla comunità, abita due mondi, senza appartenere a nessuno dei due. La sua uccisione non costituisce omicidio. Ugualmente, il bandito guatemalteco, il pandillero contemporaneo, vive al limite della società, marginalizzato da politiche segreganti. La sua uccisione non preoccupa il potere, ma viene generalmente vista come una morte cercata, meritata, inevitabile. La figura del bandito, come quella dell'homo sacer, è una soglia d'indifferenza, tra l'esclusione e l'inclusione. La figura del bandito, come l'homo sacer, mette in luce la struttura del dominio, la sua radice ed essenza, a prescindere dalla sua specifica attuazione (sia essa un regime totalitario o democratico).

La poca attenzione politica data in Guatemala allinvestigazione delle morti di giovani banditi non identificati rischia quindi di assolvere, su un piano differente, alla

39 REMHI, Guatemala nunca más: informe del proyecto interdiocesano de recuperación de la memoria histórica, Guatemala, ODHAG, 1998. Le violazioni segnalate riguardano: morte diretta, morte indiretta, sparizione forzata, sequestro, tortura, violenza sessuale, attentati, minacce, detenzione irregolare. Le organizzazioni guerrigliere sono segnalate come responsabili di 2.523 violazioni, ossia il $4,81 \%$ del totale.

40 AGAMBEN, Giorgio, Homo sacer: il potere sovrano e la nuda vita, Torino, Einuadi, 1995, p. 116. 
stessa funzione delle sparizioni forzate del periodo del conflitto armato interno: celare ciò che risulterebbe sconveniente mostrare, la natura intima del potere.

In quest'ottica anche la frase del diacono C. citata all'inizio di questo articolo può assumere allora un altro significato. «Dal punto di vista dell'antropologo»41, scrivere la violenza partendo dal luogo in cui vengono seppelliti molti pandilleros non identificati non costituirebbe un atto "pornografico», una descrizione fine a se stessa di alcuni eventi brutali, una semplice riproposizione di una costruzione simbolica stigmatizzante condivisa. Non rappresenterebbe un gesto voyeuristico, funzionale solo alla perpetuazione di stereotipi. Scrivere la violenza diverrebbe, al contrario, analisi e contemporaneamente atto di critica e di denuncia sociale ${ }^{42}$.

Il fucilato-ma-non-assassinato del conflitto armato guatemalteco e il bandito contemporaneo esplicitano una struttura originaria del potere. La loro occultazione, la loro tramutazione in $\mathrm{XX}$, in morti senza nome, è funzionale al suo nascondimento. Ricomporre l'identità di quei resti dimenticati, a partire anche da un'investigazione sociale, rappresenta allora un'azione critica, finalizzata allo smascheramento di ciò che volutamente è stato e continua oggi ad essere coperto da una coltre di silenzio, di segretezza, di terrore.

41 MALIGHETTI, Roberto, «Dal punto di vista dell'antropologo. L'etnografia del lavoro antropologico», in FABIETTI, Ugo, Etnografie e culture. Antropologi, informatori e politiche dell'identità, Roma, Carocci, 1997, pp. 201-215.

42 BOURGOIS, Philippe, In Search of Respect: Selling Crack in El Barrio, Cambridge, Cambridge University Press, 1995. 


\section{* L'autore}

Dottorando in antropologia all'Università di Verona (Scuola di Dottorato di Studi Umanistici). Sta attualmente compiendo le sue ricerche a Città del Guatemala. I suoi temi d'interesse sono: potere, contro-potere ed utilizzo del territorio urbano, violenza, banditismo giovanile transnazionale (maras e pandillas).

URL: < http://studistorici.com/progett/autori/\#GrassiPaolo >

\section{Per citare questo articolo:}

GRASSI, Paolo, «ll cimitero la Verbena: cinquant'anni di violenza politica e sociale a Città del Guatemala», Diacronie. Studi di Storia Contemporanea: Le sembianze di Clio: approcci alla storia, 29/10/2011,

URL:< http://www.studistorici.com/2011/10/29/grassi_numero_8/ >

Diacronie Studi di Storia Contemporanea $\beta$ www.diacronie.it

Risorsa digitale indipendente a carattere storiografico. Uscita trimestrale. redazione.diacronie@hotmail.it

Comitato di redazione: Marco Abram - Giampaolo Amodei - Jacopo Bassi - Luca Bufarale - Alessandro Cattunar - Alice De Rensis Barbara Galimberti - Deborah Paci - Fausto Pietrancosta - Martina Sanna - Matteo Tomasoni - Luca Zuccolo 